\title{
IdeAs
}

Idées d'Amériques

18 | 2021

Frontières dans les Amériques - Intégration, sécurité et migrations

\section{La frontière? Quelle frontière ? La dynamique transnationale de l'orpaillage clandestin en Guyane française}

Fronteira? Que fronteira? A dinâmica transnacional da garimpagem de ouro na Guiana francesa

A border? What border? Transnational dynamics of illegal gold mining in French Guiana

François-Michel Le Tourneau

\section{(2) OpenEdition}

\section{Journals}

\section{Édition électronique}

URL : https://journals.openedition.org/ideas/11295

DOI : 10.4000/ideas. 11295

ISSN : 1950-5701

\section{Éditeur}

Institut des Amériques

\section{Référence électronique}

François-Michel Le Tourneau, « La frontière ? Quelle frontière ? La dynamique transnationale de I'orpaillage clandestin en Guyane française », IdeAs [En ligne], 18 | 2021, mis en ligne le 01 octobre 2021, consulté le 21 octobre 2021. URL : http://journals.openedition.org/ideas/11295 ; DOI : https:// doi.org/10.4000/ideas. 11295

Ce document a été généré automatiquement le 21 octobre 2021.

\section{cc) $(\mathrm{S} \odot$}

IdeAs - Idées d'Amériques est mis à disposition selon les termes de la licence Creative Commons Attribution - Pas d'Utilisation Commerciale - Pas de Modification 4.0 International. 


\section{La frontière? Quelle frontière? La dynamique transnationale de l'orpaillage clandestin en Guyane française}

Fronteira? Que fronteira? A dinâmica transnacional da garimpagem de ouro na Guiana francesa

A border? What border? Transnational dynamics of illegal gold mining in French Guiana

François-Michel Le Tourneau

\section{Introduction}

1 Depuis environ 25 ans, la Guyane française est le théâtre d'une activité clandestine d'extraction d'or qui ignore les frontières de ce territoire ultramarin et l'insère dans une dynamique transnationale, celle de l'orpaillage informel brésilien - le garimpo. Entre 12000 et 15000 mineurs clandestins (Heemskerk M. et al., 2021), presque tous Brésiliens $(98 \%)$ arrachent ainsi chaque année entre 5 et 10 tonnes d'or au territoire français, revendant principalement le produit au Suriname et rapatriant in fine leurs bénéfices au Brésil.

2 Système économique, social et culturel marginal (Le Tourneau F.-M., à paraitre), le garimpo se déploie de manière réticulaire sur l'ensemble de l'Amazonie. Apparu au XVIII ${ }^{\mathrm{e}}$ siècle au Brésil, l'une de ses caractéristiques est d'ignorer les limites administratives ou juridiques. Depuis près de trois siècles, les garimpeiros exploitent l'or, le diamant ou toute sorte de minerais de valeur où ils se trouvent, envahissant sans discrimination les terres publiques, les territoires amérindiens ou les aires protégées. D’abord concentrés au Brésil, les garimpeiros se sont aventurés au Suriname ou en Guyane française à partir 
des années 1990, y déployant leur système sans se soucier des autorités locales ou des cadres nationaux.

3 Les orpailleurs brésiliens ont ainsi une logique territoriale qui leur est propre et qui articule les régions d'exploitation sous la forme d'un réseau déployé, à l'échelle de l'Amazonie brésilienne et du plateau des Guyanes. Le fonctionnement de ce réseau est transnational, ou plutôt «a-national », car il considère l'espace uniquement au prisme de la localisation des ressources sans prendre en compte les frontières. Les garimpeiros établissent ainsi des flux d'information, de matériels, de richesses et de personnes entre le Brésil, la Guyane française et le Suriname, de manière à opérer efficacement quel que soit le pays dans lequel ils se trouvent. Ils approchent les différents territoires en fonction d'une grille de lecture propre qui agrège les profits potentiels et les risques liés à la pression des autorités de chaque État, réduisant de ce fait la souveraineté de ces derniers à une simple contrainte matérielle, un paramètre, parmi d'autres, à prendre en compte.

4 Fondé sur une recherche de quatre années sur le phénomène de l'orpaillage clandestin (Le Tourneau F.-M., 2020), cet article démontre comment le "système garimpeiro » approche les frontières de la Guyane française. Pour ce faire, il aborde tout d'abord l'histoire de l'exploitation de l'or en Guyane, en montrant comment les intérêts géopolitiques liés à celle-ci et les mouvements migratoires entraînés par différentes ruées ont façonné les limites de ce territoire, puis comment le «deuxième cycle de l'or », encore en cours aujourd'hui, a vu le développement de la présence clandestine des garimpeiros brésiliens. La deuxième partie s'intéresse à l'organisation, sur le terrain, des passages de frontière, en montrant comment la Guyane est pénétrée sur trois flancs : les façades fluviales du Maroni et de l'Oyapock et la façade littorale. La dernière partie replace l'exploitation clandestine de l'or de Guyane française dans son cadre régional et global pour montrer combien le système mis en place par les orpailleurs brésiliens procède d'une logique de réseau qui contrecarre la logique aréale de la souveraineté sans toutefois la menacer dans son principe.

\section{Les deux « cycles de l'or » guyanais}

L'exploitation de l'or a doublement contribué à façonner l'intérieur de la Guyane française, d'abord en étant l'un des moteurs qui ont mené à la stabilisation des frontières (au détriment des prétentions françaises) et ensuite en étant la raison de différentes vagues de peuplement ou d'occupation de son intérieur.

\section{Le premier cycle de l'or, catalyseur de la formation des frontières et du peuplement de l'intérieur}

6 La recherche de l'or de l'Eldorado a justifié de nombreuses explorations de la Guyane dès l'installation des premiers colons français au début $d u$ xvII ${ }^{e}$ siècle. Pourtant, la découverte effective des richesses aurifères sera tardive. Ce n'est qu'en 1854-1855 que le métis brésilien, Paolino, découvre les premiers gisements et que l'entrepreneur français, Félix Couy, monte une expédition avec lui pour confirmer ses trouvailles. Se met alors en route le « premier cycle de l'or », qui durera jusqu'aux années 1950. 
7 La première phase de ce cycle est dominée par des entreprises d'exploitation minière basées sur des concessions et, souvent, des souscriptions de capital levé sur le marché local ou national, à l'exemple de la Compagnie aurifère et agricole de l'Approuague ou de la société des mines de Saint Elie (Petot J., 1986 ; Mam Lam Fouck S., 1999). Les coûts de prospection étant élevés, et les coûts d'exploitation plus encore, beaucoup firent faillite rapidement.

8 L'accès à la main-d'œuvre était aussi un obstacle majeur. Très peu peuplée, la Guyane ne disposait pas d'un réservoir humain pour soutenir une ruée vers l'or sur le mode de la Californie ou du Klondike, et sa réputation d'extrême insalubrité n'incitait pas les habitants de la métropole à se lancer dans l'aventure. Ne pouvant recourir à l'esclavage, aboli peu de temps auparavant, certaines entreprises se tournèrent vers l'importation de travailleurs volontaires venus d'Inde ou d'Afrique, sans grand succès. Les travailleurs, transportés et hébergés dans des conditions très précaires, étaient peu productifs, et les conditions de travail étaient souvent inhumaines si bien que cette pratique a été interdite en 1865 pour les travailleurs venant d'Afrique et en 1878 pour ceux venant d'Inde (Mam Lam Fouck S., 1999).

entreprises minières se trouvent donc en difficulté pour se maintenir en Guyane dans le dernier quart du XIX $x^{e}$ siècle. En parallèle, de véritables ruées vers l'or se produisent sur les frontières du territoire français, d'abord dans la région du Tapanahony, affluent de la rive gauche du Maroni, situé à l'Ouest de la Guyane française, à partir de 1874, puis, dans la région de Calçoene, située au sud du fleuve Oyapock, dans l'État brésilien d'Amapá, à partir de 1893.

10 Dans les deux cas, l'attrait de l'or a entraîné un regain d'intérêt pour la définition des frontières entre les États concernés. Dans le cas du Tapanahony, la zone était disputée entre la France et la Hollande du fait d'une incertitude sur le cours supérieur du Maroni. L'arbitrage du Tsar de Russie, délivré en 1891, considéra la Lawa comme le tributaire principal, attribuant ainsi la zone disputée à la Hollande et fixant presque définitivement la frontière ouest de la Guyane française (Blancodini, 2019). Dans le cas du Calçoene, après des escarmouches entre Français et Brésiliens en 1895, un arbitrage international est sollicité en 1899, cette fois-ci auprès de la Suisse. Rendu l'année d'après, il donne principalement raison au Brésil et fixe la frontière Est de la Guyane sur le fleuve Oyapock (Le Tourneau F.-M. et A. Greissing, 2013 ; Granger S., 2012).

11 Si ces ruées vers l'or ont considérablement aidé à fixer les limites de la Guyane, elles ont aussi joué un rôle dans son peuplement. A la fin du XIX siècle, l'épuisement des principaux gisements de Guyane hollandaise et d'Amapá a en effet laissé une population flottante de plusieurs milliers de personnes en bordure du territoire français, où de nouvelles découvertes continuaient d'être réalisées. Habitués à vivre dans des régions isolées et peu soucieux des questions de souveraineté, ces mineurs franchirent facilement les fleuves frontières en direction des nouveaux placers. Ils le pouvaient d'autant plus facilement que la présence des autorités dans l'intérieur de la Guyane était insignifiante ${ }^{1}$.

12 Le début $\mathrm{du} \mathrm{xx}^{\mathrm{e}}$ siècle voit donc une coexistence délicate entre les entreprises formelles, peu capables de maîtriser leurs territoires, et les mineurs informels. Ceux-ci s'installaient souvent autour des concessions, soit pour prospecter sur leurs limites soit pour s'approprier l'or des chantiers légaux lors d'exploitations nocturnes. Devant cette insécurité et l'incapacité des autorités à intervenir dans des zones aussi isolées, les concessionnaires sont peu à peu passés de l'exploitation directe au commerce en optant 
pour la "mise en bricole», expression qui désigne un accord informel avec les orpailleurs clandestins à travers lequel ces derniers pouvaient extraire l'or de la concession à condition de l'échanger exclusivement au magasin installé sur place par l'entreprise concessionnaire (Petot J., 1986 ; Orru J.F., 2001 ; Mam LamFouck S., 1999).

Une population de quelques milliers d'orpailleurs s'est ainsi stabilisée dans l'intérieur de la Guyane. Souvent originaires de Sainte-Lucie et presque toujours caribéens, ils auraient été entre 5000 et 6000 vers 1900 (Strobel M.B., 2019) et jusqu'à 12000 juste avant la Première Guerre mondiale (Mam Lam Fouck S., 1999). Ils sont à la base de la formation de plusieurs gros villages comme Saül, Coco, Inipi, Saint Léon... Leur nombre décroît cependant peu après la Première Guerre mondiale car la rentabilité de l'orpaillage est faible et le développement du littoral offre des conditions de vie bien plus attractives. La production artisanale décline donc et le nombre d'orpailleurs avec. On n'en compte seulement moins de 500 au début des années 1960 (BRGM, 1995).

\section{Le $2^{\mathrm{e}}$ cycle de l'or et l'orpaillage brésilien}

14 Après les années 1950, la production d'or de Guyane est devenue presque anecdotique en dehors de quelques installations semi-industrielles comme dans la région de Sophie (Petot J., 1986 ; 1993). Ce n'est qu'à partir des années 1980 que s'ouvre le « second cycle de l'or ", impulsé par l'envolée des cours du métal précieux dans les années 1970².

Si ces circonstances amènent un retour des prospections en Guyane, les impacts se font plus particulièrement sentir dans la région voisine de l'Amazonie brésilienne (Le Tourneau, F.-M. 2019), y entrainnant le renouveau de l'orpaillage informel ou garimpo. Apparu durant la période coloniale et durement réprimé à l'époque (Salomão E., 1984), celui-ci s'était maintenu dans plusieurs régions du pays (Souza L., 1982) et il était légal sur les terres publiques fédérales depuis les réformes du gouvernement Vargas dans les années 1930. Dans les années 1950, la découverte d'importants gisements aurifères alluviaux, dans la région du Tapajós, avait déclenché un premier rush (Wanderley L., 2015). Au même moment, plusieurs milliers de mineurs informels exploitaient des gisements d'étain en Rondônia (Théry, H. 1976).

16 Il faudra toutefois attendre les années 1970-1980 pour voir se dessiner une véritable ruée vers l'or (Mathis A. et al., 1997), basée sur la conjonction de plusieurs facteurs : les politiques de développement, qui ont précipité vers l'Amazonie des cohortes de paysans sans terre et qui ont créé des axes routiers rendant la pénétration vers les zones isolées beaucoup plus facile; des progrès technologiques et des innovations locales, qui ont rendu l'orpaillage informel beaucoup plus performant; une crise économique de l'État brésilien, qui a rendu ce dernier, à la fois, beaucoup moins à même de contrôler son territoire et beaucoup plus prêt à fermer les yeux sur des activités considérées comme servant les finances du pays. Durant les années 1980, on estime que jusqu'à 400000 orpailleurs informels travaillaient dans des milliers de placers éparpillés en Amazonie et que l'économie de l'orpaillage faisait vivre directement ou indirectement près d'un million de personnes (Oliveira R., 2014).

17 Cette époque dorée s'achève au début des années 1990 quand le gouvernement brésilien renoue avec sa politique en faveur des exploitations industrielles, sources d'emplois formels et de taxes, et plus faciles à contrôler. Au même moment, le cours de l'or décline et une série de scandales et de reportages de presse insistant sur les aspects les moins avouables liés à l'orpaillage, comme la prostitution de mineures, entraîne un 
revirement de l'opinion publique du pays à son sujet. De marginal romantique, l'orpailleur devient alors aux yeux de celle-ci un criminel endurci (Tedesco M., 2015).

L'augmentation de la pression sur les orpailleurs du Brésil, qui exploitaient souvent illégalement des aires protégées ou des territoires amérindiens, les a amenés à chercher d'autres aires à prospecter. Certains d'entre eux avaient commencé à travailler au Suriname, à proximité du Maroni, durant la guerre civile, et ils avaient fait la démonstration de l'efficacité de leurs techniques (de Theije M. et M. Heemskerk, 2009). Leur nombre a considérablement augmenté durant les décennies 1990 et 2000 (de Theije M. et M. Heemskerk, 2014). En Guyane française, les orpailleurs brésiliens ont d'abord été présents sur les barges qui se sont multipliées sur les grands fleuves (Oyapock, Approuague) à la fin des années 1990, dans un contexte légal un peu flou marqué par l'existence des " autorisations personnelles minières ${ }^{3}$ " sous lesquelles se dissimulaient des associations baroques entre entrepreneurs de garimpo et résidents de Guyane (Orru J.-F., 1998). La révision du code minier en 1998 et son application aux départements d'Outremer a créé une situation bien plus rigide et précipité la plupart de ces exploitants dans l'illégalité (Farruggia F., 2019). Elle a aussi donné un cadre pour des actions de répression qui commencèrent par démanteler les villages de barges apparus sur l'Approuague ou sur l'Oyapock.

Pour autant, les orpailleurs brésiliens n'ont pas quitté la Guyane, dont ils avaient pressenti la grande richesse. Délogés des zones les plus accessibles, ils se sont éparpillés au sein de la forêt, exploitant à nouveau des zones reconnues avant eux par les orpailleurs créoles. Leur présence de plus en plus importante et les graves impacts environnementaux qui en découlent, notamment la pollution au mercure, ont alors entraîné une réaction des autorités françaises (May A., 2007 ; Le Tourneau F.-M., 2020). A partir de 2002, la gendarmerie lance les "opérations Anaconda", dont la multiplication ne permet cependant pas de juguler le phénomène.

A partir de 2008, les forces armées sont engagées en soutien des forces de sécurité intérieure dans le cadre de "l'opération Harpie». Si le changement d'échelle du dispositif permet alors d'augmenter la pression sur les orpailleurs clandestins, ceux-ci s'adaptent en permanence aux techniques employées contre eux et réussissent à se maintenir grâce à la souplesse et à l'agilité de leurs systèmes de production et de ravitaillement.

On considère aujourd'hui qu'entre 12000 et 15000 orpailleurs clandestins (Heemskerk M. et al., 2021), presque tous des Brésiliens, opèrent entre 500 et 700 chantiers clandestins dans l'intérieur de la Guyane et produisent entre 5 et 10 tonnes d'or chaque année. Bien que située sur le territoire français, leur activité se joue des États. Elle déploie un système territorial dans lequel les frontières ne sont pas des barrières infranchissables mais uniquement des obligations de contournement (Oliveira R., 2014).

\section{Les flux d'orpaillage et les frontières de la Guyane}

L'activité des garimpeiros en Guyane repose sur des franchissements constants de frontière, qui s'exercent sur trois des bordures de la Guyane française: les façades fluviales de l'Oyapock et du Maroni, et la façade littorale (Figure 1). 


\section{La façade de l'Oyapock}

La frontière est de la Guyane a été l'une des zones les plus actives au début de la montée de la présence des orpailleurs brésiliens en Guyane et la ville d'Oiapoque a vu son activité et son économie tourner principalement autour du garimpo durant les décennies 1990 et 2000. Directement concernée par la flambée de l'exploitation des barges d'orpaillage sur le fleuve Oyapock, elle a ensuite été la plaque tournante pour les garimpeiros qui remontaient le fleuve- dont la souveraineté est partagée entre la France et le Brésil - pour pénétrer vers l'intérieur du territoire français. Bien que moins importants aujourd'hui, les flux y restent significatifs, Oiapoque demeurant l'une des portes d'entrée pour l'orpaillage clandestin en Guyane française.

L'organisation des orpailleurs sur cette façade s'appuie sur trois points principaux. Le premier est la ville d'Oiapoque. Bien que le centre de gravité des activités se soit déplacé vers le Maroni, elle supporte encore la logistique des chantiers des régions de l'est de la Guyane (Ouanary, Sikini, Approuague). Elle constitue aussi une zone de transit pour celles et ceux qui ne disposent pas d'un passeport leur permettant de se rendre directement par avion au Suriname en proposant deux alternatives: soit embarquer sur une des pirogues effectuant la liaison maritime clandestine vers Albina (voir II.3), soit remonter l'Oyapock et suivre la piste qui borde la rivière Sikini. Dans ce cas, il leur faudra cheminer à pied entre une et deux semaines pour parvenir à la région de leur choix. Ces itinéraires fonctionnent dans les deux sens. Oiapoque est ainsi également le point d'arrivée de ceux qui rentrent au pays, temporairement ou définitivement, et qui ne peuvent prendre l'avion soit parce qu'ils n'ont pas assez d'argent, soit parce que, n'ayant pas de papiers, ils ne peuvent se régulariser au Suriname ${ }^{4}$.

Plus en amont sur l'Oyapock, deux villages appuient les activités d'orpaillage. Le premier est Ilha Bela, situé sur l'île du même nom, en face de la rivière Sikini. Entièrement dédié au garimpo, ce village est une base arrière dans laquelle sont stockés vivres et matériels attendant leur embarquement pour l'intérieur de la Guyane française. C'est aussi le point de départ pour les travailleurs et les travailleuses qui veulent tenter leur chance "en France ». Malgré la présence du poste de contrôle de Carbet-Légion, situé à l'embouchure de la Sikini, de nombreux itinéraires de contournement permettent de passer vers l'amont de la rivière puis de gagner le centre de la Guyane, à partir d'où on peut rejoindre n'importe quelle région exploitée par les orpailleurs. 
Figure 1 : les points d'entrée de l'orpaillage clandestin en Guyane

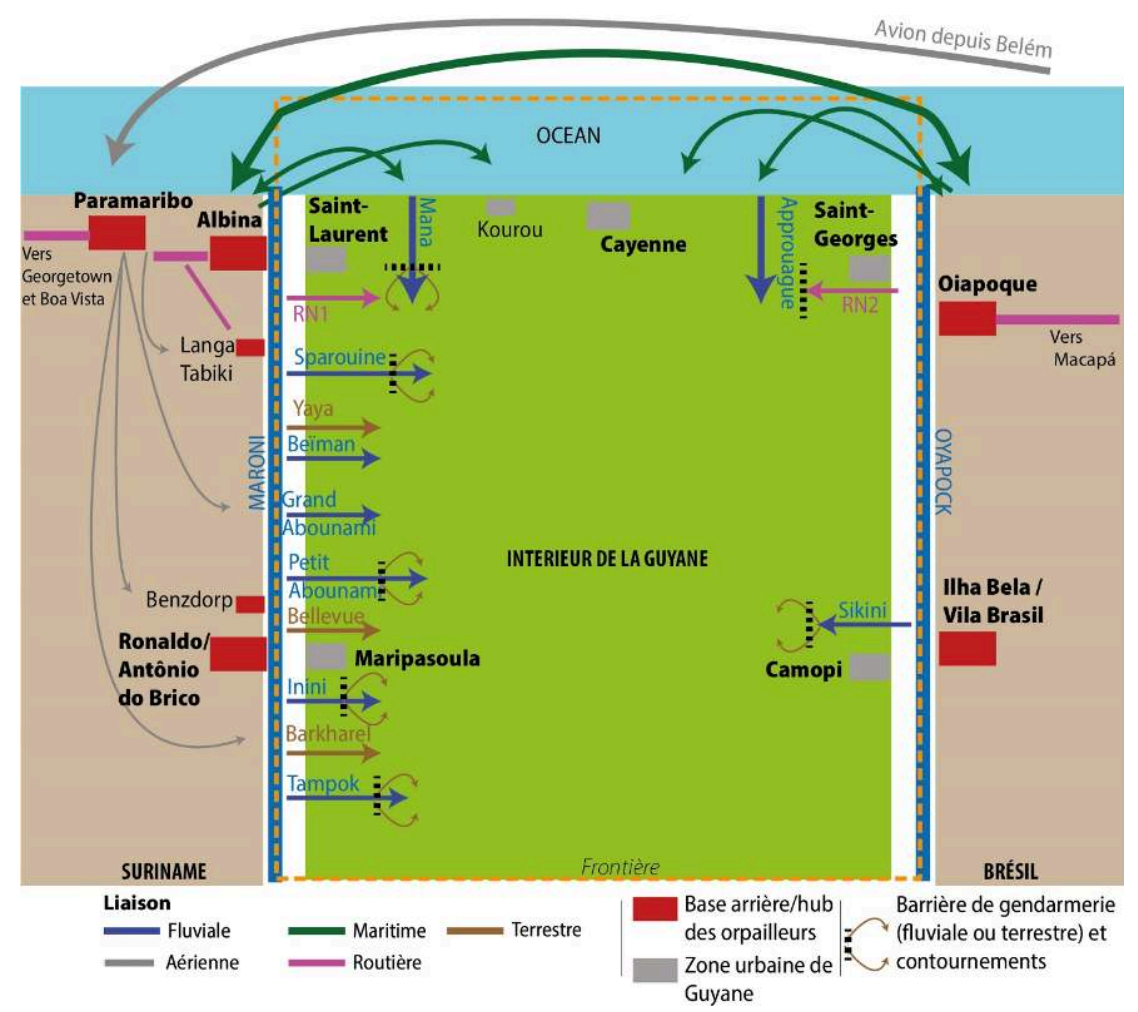

Un peu plus haut sur le fleuve, Vila Brasil dispose d'un semblant d'officialité dont ne jouit pas Ilha Bela, même si son inclusion dans un parc national, au sein duquel les activités humaines sont en principe interdites, reste sujette à débat (Jacob T., 2018). Le village vit autant de l'orpaillage que du commerce avec la population amérindienne de Camopi, bourg situé juste en face sur la rive française, qu'il approvisionne à des prix plus bas que les épiceries de ce dernier.

La manière dont la frontière de l'Oyapock se présente est différente pour les habitants des villes jumelles d'Oiapoque et de Saint-Georges de l'Oyapock et pour les orpailleurs. Pour les premiers (et, d'une manière générale, pour les habitants du littoral guyanais), la limite entre la France et le Brésil génère les effets d'opportunité et les phénomènes que l'on retrouve couramment en contexte transfrontalier. Les prix plus avantageux des biens et des services au Brésil entraînent des flux d'acheteurs de la Guyane française vers ce dernier, alors que le niveau de vie supérieur côté français entraîne des flux de travailleurs en sens inverse. Dans les deux cas, la construction du pont sur l'Oyapock a eu un effet paradoxal, puisque, alors que l'objectif était de favoriser la communication, elle a au contraire entraîné une rigidification de la frontière motivée par la peur d'une migration massive de Brésiliens en direction de Cayenne (Silva G. et al., 2020).

Pour les orpailleurs, la frontière de l'Oyapock ne constitue pas en elle-même un élément bloquant pour les activités. En effet, en dépit d'un traité de lutte contre l'orpaillage illégal signé par les deux pays en 2008, les contrôles sont infiniment moins probables du côté du Brésil. Les pirogues qui remontent l'Oyapock restent donc le plus possible dans la moitié brésilienne du fleuve pour éviter les interceptions, avant de décharger leur fret à Vila Brasil ou Ilha Bela, d'où il sera acheminé vers l'intérieur de la Guyane dès qu'une occasion favorable se présentera. L'ouverture du pont sur l'Oyapock, 
elle, a eu pour effet que les garimpeiros utilisent désormais moins la route RN2 que par le passé car elle est étroitement surveillée par la gendarmerie qui maintient un checkpoint sur le pont de Régina. Les candidats à l'orpaillage passent donc soit par la route de la Sikini, au sud, et, de plus en plus, par la liaison maritime clandestine en direction d'Albina.

\section{La façade du Maroni} basculement progressif des activités d'orpaillage vers l'ouest de la Guyane, particulièrement manifeste depuis une douzaine d'années. A cette raison logistique (les flux de ravitaillement utilisent principalement le réseau fluvial et donc les affluents de rive droite du Maroni sont autant de voies de communication vers les zones exploitées) s'ajoutent le fait que l'orpaillage est légal ou toléré ${ }^{5}$ au Suriname et que la vente de l'or y est facile, les comptoirs d'achat considérant que tout l'or qui leur est apporté est produit légalement sur place. Ces comptoirs proposent aussi des facilités de virement bancaire permettant aux orpailleurs de rapatrier leur argent au Brésil (voir III.1). Enfin, la croissance continue de l'activité a entraîné l'installation d'un réseau de commerçants chinois spécialisés dans le ravitaillement des sites d'orpaillage. De la batée ${ }^{6}$ au quad en passant par les produits alimentaires fabriqués au Brésil, ils proposent tout le nécessaire pour approvisionner les chantiers sans avoir besoin d'aller plus loin que la rive gauche du Maroni. jusqu'à Langa Tabiki environ, est polarisé par la ville d'Albina, dans laquelle dominent les populations bushinenguées ${ }^{7}$, mais dans laquelle il existe aussi une population brésilienne liée à l'orpaillage, concentrée autour des entrepôts du supermarché Transamérica. La ville ravitaille directement les zones d'orpaillage du bas Maroni (tant du côté français que du côté surinamais) ou celles de la Mana. Elle est aussi le point de départ de pirogues transportant des gros volumes en direction des tronçons situés plus en amont. Elle est enfin le point d'arrivée de la liaison maritime qui la relie à Oiapoque. Mais si Albina domine sur ce tronçon, d'autres nœuds logistiques existent, notamment à proximité de l'île de LangaTabiki, qui dispose d'un aérodrome et d'un accès routier sur la rive surinamaise.

31 Le deuxième tronçon recouvre le cours moyen du Maroni, de Langa Tabiki à Benzdorp. Il est émaillé de quelques villages et de quelques commerçants chinois installés en face de chaque affluent du Maroni qui s'enfonce dans le territoire guyanais comme les criques Sparouine, Beïman ou Petit Abounami, etc. Ce tronçon dispose aussi de liaisons aériennes par l'intermédiaire de plusieurs petits aérodromes. Il se termine à Benzdorp, un village correspondant à une région d'orpaillage autrefois très importante, située du côté du Suriname. Le déclin de cette dernière a entraîné un glissement des activités vers le bourg de Ronaldo.

tronçon se situe sur le haut Maroni (appelé la Lawa en amont de la confluence avec le Tapanahoni), entre Maripasoula et la confluence entre Litani et Marouini. Il est principalement marqué, côté surinamais, par la présence d'un doublon de villages qui rappelle ceux du haut-Oyapock. Petit-Albina (ou Albina 2, ou New Albina), appelé Antônio do Brinco par les orpailleurs, se trouve juste en face de la ville française de Maripasoula. Principalement né du soutien aux activités d'orpaillage, ce 
village a un semblant de reconnaissance par les autorités du Suriname, notamment du fait de la présence d'un poste de police. Il concentre plusieurs entrepôts chinois. A quelques centaines de mètres en amont se trouve un autre hameau, dénommé Ronaldo. Initié par un commerçant péruvien (d'où son premier nom de Peruano), bientôt remplacé par un Brésilien (Ronaldo), lui-même évincé par des commerçants chinois, il ne vit que pour l'orpaillage. Les entrepreneurs chinois y possèdent à peu près tous les bâtiments et ils déploient des activités diversifiées. Autour d'un entrepôt-supermarché, ils proposent en général aussi des chambres d'hôtel, des maisons à louer, et ils laissent les orpailleurs de passage installer leurs hamacs sous leurs entrepôts, où se situent aussi des échoppes qu'ils louent pour toute sorte de services (coiffeurs, magasins d'articles de mode, de fruits et légumes frais, etc.). A Ronaldo comme à Petit-Albina, on trouve toute la gamme des infrastructures d'appui à l'orpaillage, des ateliers mécaniques aux supermarchés, et des salons de beauté aux maisons closes, en passant par les night clubs.

33 Le Maroni est une artère fluviale très active malgré les difficultés qu'il pose en saison sèche. Des pirogues chargées de dizaines de fûts de carburant, de provisions, de matériel d'orpaillage ou encore de travailleurs et de travailleuses le montent ou le descendent constamment. Comme sur l'Oyapock, elles se maintiennent hors des eaux françaises pour ne pas être interceptées.

La navigation sur le Maroni ou sur ses affluents situés au Suriname est en général effectuée par des piroguiers bushinengués. Celle sur les eaux des rivières françaises qui ne sont pas barrées par la gendarmerie est le plus souvent le fait de Brésiliens qui utilisent des embarcations plus petites et plus rapides. Quand les cours d'eau sont barrés (Inini, Abounami, Tampok...) les piroguiers délivrent leurs marchandises à l'entrée de chemins de contournement, d'autres pirogues venant en amont pour récupérer les charges et continuer le voyage. Tout un univers de porteurs et de pilotes de quad vit donc à proximité de ces points de passage, gagnant leur vie en réalisant les contournements. Le port d'une charge de $50 \mathrm{~kg}$ sur deux ou trois kilomètres rapporte de 80 à $150 €$ en fonction de la difficulté du trajet, une rotation de quad avec 200 à 250 $\mathrm{kg}$ de charge sur une vingtaine de kilomètres revenant à $600 €$. Un porteur à pied qui réalise trois navettes en une nuit ou un pilote de quad effectuant un trajet peuvent ainsi gagner un salaire confortable.

Au contraire de l'Oyapock, sur lequel l'activité des barges d'orpaillage est interdite tant du côté français que du côté brésilien, le Maroni est ouvert à l'exploitation du côté surinamais. Pendant longtemps, des barges tentant de travailler au plus près de la frontière ont entraîné des frictions entre le gouvernement français et le gouvernement surinamais. La signature en février 2021 d'un accord technique sur le tracé de la frontière sur le Maroni et la Lawa, et sur la souveraineté sur chacune des îles qui s'y trouvent (dont la délimitation était délicate du fait de la dynamique fluviale dans laquelle les îles peuvent se déplacer), devrait désormais résoudre cette question ${ }^{8}$.

Comme l'Oyapock, du point de vue des orpailleurs brésiliens, le Maroni n'est pas une barrière. Il est au contraire une artère de communication essentielle et relativement sûre à partir de laquelle il est possible à tout moment de bifurquer en direction de zones d'exploitation situées en territoire français. Pour les autorités françaises, le défi est presque impossible : comment empêcher les passages de frontière sur une ligne fluviale de près de 350 kilomètres de long, où il est possible de débarquer à n'importe quel point pour se perdre dans la forêt? Dans certains cas, des pistes informelles (piste 
Bellevue, piste Yaya, piste Barkharel) relient les berges du Maroni à l'intérieur de la Guyane, déjouant la surveillance mise en place sur les voies fluviales.

\section{La façade littorale}

Le littoral de la Guyane est lui aussi utilisé par les orpailleurs clandestins. Son premier usage est le contournement du territoire français par la mer. Violant allégrement les eaux territoriales françaises, la liaison maritime clandestine entre Albina et Oiapoque achemine principalement des personnes, soit du Brésil en direction des zones de travail du Suriname et de l'ouest de la Guyane française, soit du Suriname vers le Brésil pour ceux qui rentrent au pays. Elle met donc en relation les interfaces qui existent à l'est et à l'ouest entre la Guyane et le garimpo. Certaines marchandises de valeur peuvent aussi être acheminées: pirogues en aluminium fabriquées au Brésil et envoyées vers le Suriname, quads importés au Suriname et envoyés vers les zones d'orpaillage de l'est de la Guyane... La liaison Albina-Oiapoque sert également au trafic de stupéfiants (et parfois d'armes) dont le Suriname est une plaque tournante.

Deux fleuves de Guyane sont aussi utilisés par les orpailleurs à partir de la façade littorale. Le premier est l'Approuague. Des pirogues de grande taille relient Oiapoque aux premiers grands sauts de ce fleuve, où des pirogues plus petites viennent récupérer les charges pour les dispatcher en direction de l'amont et de zones comme Grande Usine, Sapokaï, Carbet Mitan, etc. A l'ouest, une situation équivalente existait sur la Mana, ravitaillée à partir d'Albina. L'installation d'une barrière de gendarmerie à Saut Valentin a cependant modifié l'organisation de ce trajet. Les marchandises partent aujourd'hui plus souvent d'Albina en direction de Saint-Laurent puis de la RN1. Après un contournement terrestre de la barrière, elles remontent la Mana en direction de l'amont. Permettant le ravitaillement de zones d'orpaillage comme les secteurs Kokioko, Délices, Sophie-Dagobert et bien d'autres, la Mana est une autre artère fluviale majeure.

Finalement, la façade littorale est aussi utilisée pour ravitailler la région du lac de Saint-Elie via l'Iracoubo ou la Sinnamary, ou encore pour débarquer sur des plages à proximité de Cayenne ou de Kourou. Dans ce dernier cas, les liaisons ne sont pas propres à la logistique de l'orpaillage. Il s'agit de pirogues soutenant la contrebande et l'immigration irrégulière vers les zones littorales de la Guyane, dont se servent à l'occasion les orpailleurs.

\section{Traversée de la frontière et calcul bénéfice / risque}

Qu'il s'agisse de la façade littorale ou des façades fluviales, l'extension des zones à contrôler et les limitations des moyens des forces de sécurité font que le contrôle, loin d'être inexistant, se concentre sur certains points considérés comme vitaux (barrières fluviales de la Mana, de l'Inini, de la Sikini, etc. ; barrière terrestre de Régina, points de contrôle à Saint-Laurent ou Saint Georges, etc.). Des patrouilles régulières tentent de rendre plus difficile l'entrée des marchandises et des personnes, mais elles ne peuvent interrompre totalement les flux. Dès lors, du point de vue des orpailleurs, toute traversée de la frontière devient un simple calcul de bénéfice/risque entre la possibilité d'être intercepté et de perdre son investissement et le gain potentiel si on parvient à bon port. Un des effets paradoxaux des contrôles est que plus ceux-ci sont efficaces, 
plus le prix des marchandises augmente dans les zones d'exploitation, rendant l'aventure encore plus rentable en cas de succès. Les chantiers d'orpaillage payent ainsi entre 5 et $10 €$ le litre de carburant quand celui-ci est acheté pour moins de $0,5 €$ à Paramaribo ou Albina, et jusqu'à $10 €$ le kilo de riz quand celui-ci revient difficilement à plus de $1 €$ chez les commerçants chinois. De tels tarifs permettent de continuer à gagner de l'argent même en perdant une cargaison sur deux ou trois...

\section{Système transnational ou a-national ?}

41 L'action des orpailleurs clandestins repose sur des communications clandestines entre l'espace du département français et d'autres régions situées à proximité, comme le Brésil et le Suriname, mais aussi à grande distance, comme celles où sont produits les moteurs ou celles vers lesquelles l'or est finalement exporté. Cette logique réticulaire des garimpeiros contredit la logique aréale de la souveraineté des États, mais elle ne s'y oppose pas frontalement car les orpailleurs clandestins voient leur présence comme temporaire.

\section{A qui profite l'or de Guyane?}

Regarder les flux économiques qui structurent l'orpaillage clandestin (figure 2) permet de voir comment celui-ci efface en quelque sorte les frontières pour mettre en connexion des espaces parfois très éloignés.

L'or est la monnaie d'échange dans le monde de l'orpaillage. Les ouvriers dépensent ainsi une grande partie de leurs gains en loisirs ou produits de première nécessité, et les patrons de chantier doivent soit acheter aux commerçants tous les produits nécessaires à la bonne marche de leur affaire, soit payer les logisticiens pour leur acheminement. Tous s'approvisionnent auprès de supermarchés (chinois sur la façade du Maroni), payant souvent leurs achats en métal précieux. L'or produit change donc souvent plusieurs fois de mains avant de parvenir aux comptoirs d'achat (compra de ouro) où tous les acteurs du garimpo, des simples ouvriers aux commerçants chinois en passant par les patrons de chantier, viennent finalement vendre leur produit.

La plus grande partie de l'or extrait en Guyane française est écoulée au Suriname. Il y est apporté en petites quantités par les travailleurs du garimpo, qui profitent de chacun de leurs passages en ville pour apporter non seulement les quelques dizaines de grammes qu'ils ont économisés eux-mêmes, mais aussi ceux confiés par toutes les personnes qui leur font confiance pour vendre leur production et faire parvenir l'argent à leur famille. L'or est aussi apporté par les patrons, en plus grande quantité et en général le plus discrètement possible pour éviter les vols à main armée. Les comptoirs d'achat rémunèrent le vendeur en dollars américains, convertibles sur place en dollars surinamais, mais aussi en reais brésiliens par l'intermédiaire de virements bancaires 9 sur le compte du bénéficiaire au Brésil. C'est par ce biais que de nombreux travailleurs et travailleuses de l'orpaillage subviennent aux besoins de leurs familles. Lorsque l'or est écoulé au Brésil, en général à Oiapoque, les opérations bancaires sont encore plus simples. Les comptoirs d'achat y sont cependant périodiquement surveillés par la police fédérale car l'orpaillage n'est pas légal partout au Brésil. Les comptoirs doivent donc disposer d'une double comptabilité, ou bien justifier leurs achats par la 
présence à proximité de zones d'orpaillage légal dont l'or est censé provenir. Dans le cas d'Oiapoque, les zones d'orpaillage du Calçoene peuvent jouer ce rôle.

Figure 2 : I'orpaillage clandestin en Guyane, flux et frontière

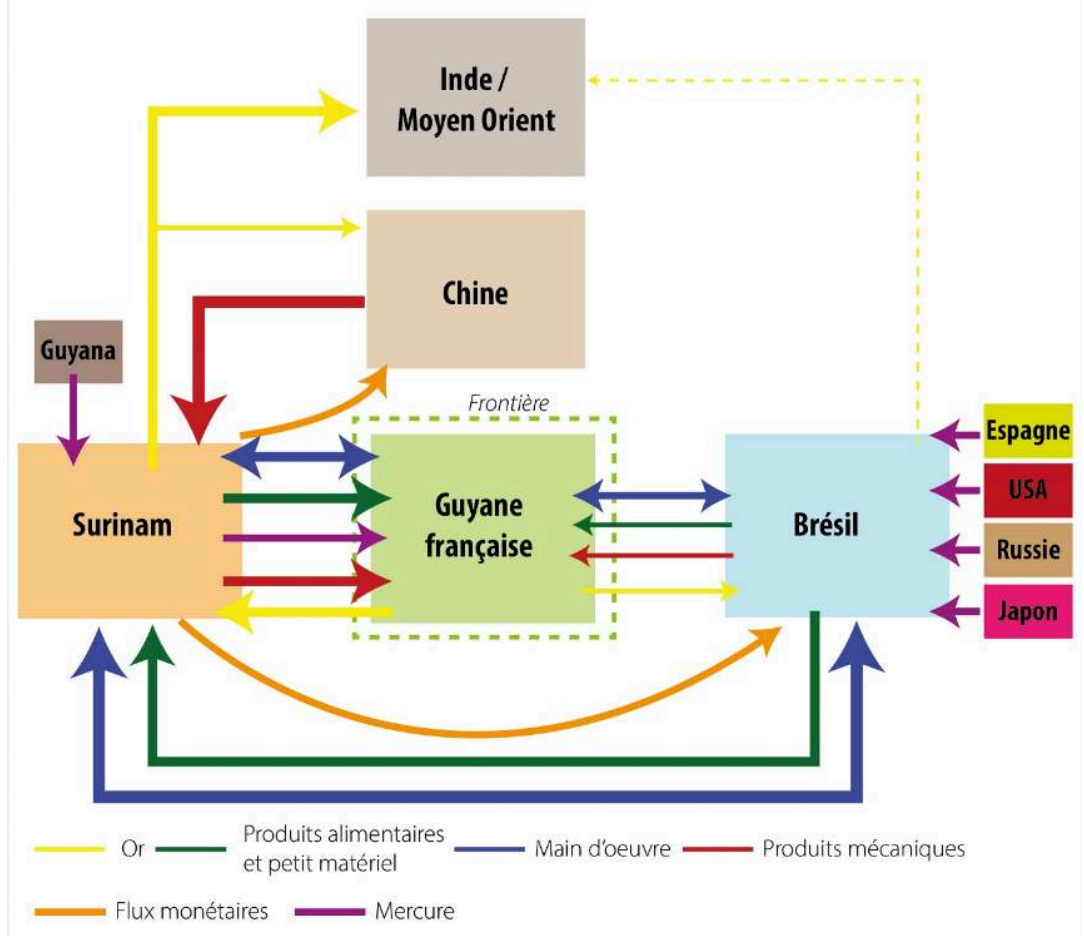

Une autre partie des bénéfices est réalisée par les commerçants situés dans les bases arrière et par leurs fournisseurs, qui sont donc des bénéficiaires lointains de l'or de Guyane. Sur la façade Maroni, Les commerçants chinois importent des produits alimentaires et des produits courants depuis le Brésil afin que les marchandises correspondent au goût de leur clientèle. Pour les matériels d'exploitation, les éléments les plus simples (tuyaux, becs de jet, corps de pompe, batées, etc.) sont eux aussi souvent importés depuis le Brésil. Pour ce qui est des éléments mécaniques (motopompes, quads, groupes électrogènes, etc.), les commerçants chinois proposent parfois des produits de qualité, de marque japonaise, américaine ou européenne, mais surtout des matériels peu chers fabriqués en Chine (leur destruction à court ou moyen terme étant quasiment inéluctable, les orpailleurs considèrent souvent leur matériel comme du consommable). Ces importations très bon marché permettent aux garimpeiros de diminuer leurs coûts fixes et d'absorber les impacts de la répression. Elles mettent aussi en contact le monde du garimpo et le complexe industriel asiatique par l'intermédiaire de la diaspora chinoise. Du côté du Brésil, les matériels vendus sont principalement de marque brésilienne, et ils sont plus chers à l'achat que les équivalents chinois. Dans certains cas, comme pour les quads, faire venir des machines depuis le Suriname peut s'avérer plus rentable.

L'insertion de l'or produit clandestinement en Guyane dans l'économie globale se réalise aussi en bout de chaîne, lorsque l'or est purifié et inséré sur le marché international. Au Suriname, la raffinerie du groupe Kaloti est la seule capable de produire de l'or 24 carats à destination des marchés les plus demandeurs (Moyen- 
Orient, Inde et Chine). Une partie de l'or est aussi probablement exportée en contrebande vers les États-Unis et là encore insérée dans le marché international. Au Brésil, l'or provenant de Guyane française est inséré dans le circuit local, lui aussi connecté, légalement ou non, avec les marchés internationaux. Il est cependant impossible de dire quelle proportion de l'or guyanais vendu au Brésil est effectivement exportée vers l'étranger.

De faible volume et aisé à dissimuler, l'or extrait illégalement de Guyane française passe les frontières du département français pour s'insérer dans l'économie régionale ou globale, que ce soit sous la forme d'or pur destiné au marché international ou sous la forme de bénéfices retirés en monnaie par les orpailleurs et réinvestis dans leur vie au Brésil et, de plus en plus, au Suriname. Le mercure, utilisé pour extraire l'or plus facilement, circule en sens inverse. Il est en effet produit dans un nombre restreint de pays (Figure 2) et entre en contrebande en Guyane française via le Suriname ou le Brésil avec l'ensemble des autres ingrédients. Mercure et or sont donc deux produits qui utilisent symétriquement l'effacement contemporain des frontières et l'augmentation des échanges commerciaux (ou, pour aller vite, la globalisation de l'économie et ses conséquences) pour pénétrer en Guyane.

\section{La logique réticulaire et continentale de l'orpaillage face à la logique aréale des territoires nationaux} Guyane française provient intégralement du Brésil, à l'instar de $98 \%$ de la population qui y est impliquée ${ }^{10}$. Plus que le reflet d'un débordement du phénomène depuis le Brésil et en direction du plateau des Guyanes, ce fait illustre la logique réticulaire et continentale de monde du garimpo.

Du fait de la géographie des dépôts aurifères, les zones d'orpaillage ne forment en effet jamais des grandes régions continues. Elles apparaissent au fur et à mesure des découvertes, disparaissent lorsqu'elles semblent épuisées (parfois avant qu'une nouvelle découverte ne les relance), et forment une sorte de semis irrégulier qui couvre l'ensemble du bassin amazonien à la manière des tâches d'une peau de jaguar. Prise isolément, chaque zone a sa dynamique propre et forme un système complet associant production, approvisionnement des chantiers et exportation de la production. Ces zones sont physiquement isolées les unes des autres et souvent difficiles d'accès, les orpailleurs ayant beaucoup plus de mal à disputer à des entreprises formelles des dépôts facilement accessibles.

Mais bien que chaque région soit une entité à part, le monde du garimpo est unifié par la circulation de flux. Les flux d'information relient ainsi l'ensemble des zones les unes avec les autres. Les nouvelles plus importantes sont les fofocas, un mot qui désigne la rumeur en portugais mais qui prend un sens tout à fait particulier dans le monde de l'orpaillage. La fofoca désigne la découverte de gisements importants et la ruée vers l'or, petite ou grande, qui la suit nécessairement. Les indications sur les «bons coins » et sur les itinéraires pour y parvenir, sur les conditions locales et sur les dangers éventuels, étaient auparavant intensément échangées sur les réseaux radiophoniques que les garimpeiros mettaient en place et qui leur permettaient parfois de communiquer avec des collègues situés à plusieurs centaines voire à des milliers de kilomètres. Aujourd'hui, les nouvelles sont échangées par Internet, en particulier par 
l'intermédiaire d'applications comme WhatsApp. Extrêmement mobiles, les garimpeiros cultivent leurs réseaux de relations car ce sont eux qui garantissent l'accès aux bons plans, la solidarité et, souvent, la sécurité.

51 Ces informations orientent les flux de personnes. Chaque orpailleur veut maximiser ses chances de s'enrichir et il recherchera les zones qui lui semblent les plus dynamiques en fonction des nouvelles reçues - qui ne sont pas toujours fiables. Mais d'autres éléments entreront aussi en ligne de compte, qui ramènent aux contextes nationaux. Informel ou clandestin, le garimpo est toujours sous la pression des autorités. Les modes d'action de celles-ci et les risques éventuels qui y sont associés sont attentivement étudiés et commentés. Ainsi, les orpailleurs actifs en Guyane française reconnaissent que l'action des forces de police y implique un risque important de perte de capital, mais ils ajoutent qu'elles ne représentent pas un risque physique pour les individus, au contraire du Brésil où la police est connue pour avoir la main lourde. Cette différence explique en partie le choix du territoire français pour ceux qui s'y trouvent. Le risque personnel est considéré encore moindre au Suriname ${ }^{11}$, mais les bénéfices y sont vus comme moindres également car les gisements sont moins riches et du fait des accords informels nécessaires pour pouvoir les exploiter qui impliquent de payer au moins $10 \%$ de la production aux leaders des communautés locales, réduisant le gain d'autant. Le Guyana ou le Venezuela sont vus comme des zones plus volatiles, du fait de la corruption des autorités et la possibilité de violences. Dans cette espèce de "géopolitique du garimpo", la Guyane apparaît comme une zone paradoxalement attractive malgré les efforts des autorités pour la rendre dissuasive.

52 La nature discontinue des zones d'orpaillage et les flux qui les unissent révèlent la nature réticulaire et transfrontalière du monde du garimpo. Celui-ci est composé d'une série des nœuds (gisements et régions exploités, bases arrière logistiques, etc.) et de relations entre ceux-ci qui permettent les échanges d'information et les mouvements des personnes. Ce réseau (ou filet, pour revenir à l'étymologie latine de rete) se déploie sur l'ensemble de l'Amazonie brésilienne et il a facilement intégré la Guyane française et le Suriname à partir des années 1990, ce qui lui donne donc une dimension transnationale. Peu concerné par les limites administratives - hors du fait qu'elles posent des difficultés logistiques qui font monter les coûts d'exploitation et, en conséquence, le prix des marchandises - ce réseau contredit à l'évidence la logique des États, qui est, elle, fondée sur le contrôle d'aires continues.

\section{Des acteurs a-territoriaux}

53 Malgré cette contradiction, l'activité des orpailleurs clandestins en Guyane française, pour irritante qu'elle soit aux yeux des autorités, est plus mise en cause pour ses impacts environnementaux (déforestation et pollution par le mercure principalement) que pour ses éventuelles implications géopolitiques, ce alors que les zones qu'ils occupent au sein de la forêt sont des enclaves dans lesquelles, de la langue aux produits alimentaires en passant par les personnes, tout provient du Brésil. La raison se trouve probablement dans l'absence de revendication politique de la part des garimpeiros. Ceux-ci s'intéressent uniquement à l'or et voient toujours leur présence comme temporaire, destinée à durer uniquement autant que les gisements fourniront assez pour que les chantiers soient rentables. Ils ne sont donc pas vraiment des migrants, et 
encore moins des envahisseurs puisque leurs plans pour le futur impliquent invariablement un retour - de préférence triomphant - vers leur terre natale.

De manière assez surprenante, les garimpeiros ne nient jamais l'illégalité de leur présence ou de leur action. Ne " pas être au bon endroit » (estar no lugar errado) est un point qu'ils concèdent facilement. Les incidents dans lesquels ils s'opposent aux actions destinées à les déloger sont très rares et ils dégénèrent peu souvent en affrontements ${ }^{12}$. Le plus souvent les orpailleurs acceptent la répression avec fatalité, considérant que les gendarmes et les militaires «ne font que leur travail» en détruisant leurs installations. Pour autant, ils quittent rarement la Guyane après ce genre d'épisode. Ils font le gros dos puis remettent peu à peu leurs chantiers en route, espérant que l'opération suivante ne vienne pas trop vite.

Dans leur logique, les orpailleurs opposent à la logique juridique de leur illégalité deux éléments qui leur semblent d'une valeur supérieure. Le premier est leur droit à la survie. Selon eux, les conditions qu'ils rencontrent au Brésil ne leur donnent pas de vraie chance de s'élever dans la société. Ils viennent donc chercher cette chance en Guyane française, et ils l'obtiennent par un dur labeur et par l'affrontement des rudes conditions de vie de la forêt. Cette logique de survie leur semble non pas totalement annuler l'illégalité de leur présence, mais au moins l'amenuiser. Le second élément est en quelque sorte l'éthique de leur profession. L'office de l'orpailleur, son talent, est de trouver l'or et de l'extraire, où qu'il se trouve et indépendamment des frontières ou des limites quelles qu'elles soient (et souvent en dépit de ces éléments). Il serait en quelque-sorte immoral de laisser cette richesse capable de transformer la vie de tant de personnes, dormir sous la terre. On peut dire sans exagérer que l'idée de réserves inexploitées est aussi intolérable au garimpeiro que l'idée d'un matelas de billets dormant chez un particulier pour un banquier. Ici encore, aux yeux des garimpeiros, la logique de leur office n'annule pas la gravité de leurs infractions mais elle la diminue. Péché véniel, donc, expié par les pertes répétées de capital et de matériel.

Ces différents éléments nourrissent une revendication profonde du monde de l'orpaillage, qui souligne que «les garimpeiros sont des travailleurs (œuvrant éventuellement au mauvais endroit et éventuellement en infraction de nombreuses lois) mais ils ne sont pas des criminels ». Ils acceptent donc comme "faisant partie du jeu » d'être expulsés ou de voir leur matériel détruit, mais ils considèrent comme disproportionnés et injustes les traitements plus agressifs (menottage, prise à partie, etc.). Selon cette vision, que l'on n'est évidemment pas obligé de partager, les questions de frontière sont de simples éléments de contexte qui peuvent être temporairement contournés pour répondre à des impératifs plus forts ou plus pressants.

\section{Conclusion}

57 Le système d'exploitation clandestin de l'or de Guyane française mis en place par les garimpeiros brésiliens va à l'encontre de la souveraineté de la France sur ses ressources et il viole les frontières du territoire français. Il repose sur une immigration illégale, pour les travailleurs, et sur de la contrebande dans les deux sens, du Suriname et du Brésil vers la Guyane pour les marchandises, de la Guyane vers ses voisins pour l'or. Par le système économique, culturel et social du garimpo, l'orpaillage brésilien insère la Guyane (ou plutôt diverses zones en Guyane française) dans un vaste réseau qui recouvre l'ensemble du bassin amazonien et du plateau des Guyanes, et au sein duquel 
circulent travailleurs, travailleuses, savoir-faire et informations. Par l'intermédiaire de la dynamique de globalisation commerciale qui permet les flux de marchandises ou d'intrants divers (aliments, combustible, mercure, etc.), il met également la Guyane en relation avec des espaces lointains comme la Chine, le Moyen Orient et bien d'autres pays.

La dynamique économique de l'orpaillage clandestin est donc clairement transnationale et elle a comme caractéristique de basculer dans l'illégalité à un moment de la chaîne, en général au passage d'un État à l'autre. Cela peut se produire sur les frontières de la Guyane, comme pour les moteurs venus de Chine qui cessent d'être légaux quand ils entrent en contrebande via le Maroni, ou bien se produire à grande distance, comme les importations de mercure de divers pays, qui sont détournées de leur but initial ${ }^{13}$ pour finir dans la main des orpailleurs. A l'inverse, l'or produit suit un circuit lui permettant de faire son chemin vers les marchés internationaux légaux.

Dans ce contexte, les frontières des États concernés ne sont pas vues comme des barrières. Dans la vision continentale des garimpeiros, elles délimitent des territoires aux opportunités différentes, que chacun peut choisir de fréquenter ou non en fonction de son analyse des bénéfices et des risques qu'ils impliquent. Leur valeur change aussi en fonction des fluctuations des politiques de répression visant l'orpaillage. En tout état de cause, la présence des frontières n'est pas un élément considéré comme interdisant, en soi, le passage, mais plutôt comme de simples contraintes de voyage impliquant certains aléas mais aussi des opportunités de revenus supplémentaires.

La géographie des frontières la Guyane se prête bien à ces incursions clandestines. Les deux fleuves Oyapock et Maroni proposent des centaines de portes d'entrées potentielles le long de leur cours, et la façade littorale n'est pas non plus imperméable aux pirogues. Se heurtant à cette réalité, les actions des autorités françaises pour améliorer leur contrôle se concentrent sur certains points clés et sur des actions coup de poing destinées à briser l'économie du garimpo. Bien que son effet soit loin d'être nul - les orpailleurs sont les premiers à reconnaître la pression qu'il exerce sur eux - ce dispositif n'est pas suffisant pour dissuader l'exploitation de l'or et, conséquemment, le non-respect des frontières françaises par les garimpeiros. Cette atteinte à la souveraineté est toutefois amoindrie par le fait que le « système garimpeiro » est en fait a-national sur le plan social et culturel, n'impliquant ni volonté d'installation à long terme, ni revendication politique. Bien qu'ils aient créé de véritables enclaves brésiliennes dans le centre de la Guyane, les orpailleurs clandestins ne se voient pas y rester et leurs plans, à moyen et long termes, sont toujours de revenir chez eux, au Brésil.

\section{BIBLIOGRAPHIE}

Blancodini, Patrick, «La frontière Suriname - Guyane française : géopolitique d'un tracé qui reste à fixer », Géoconfluences, septembre 2019, http://geoconfluences.ens-lyon.fr/informations- 
scientifiques/dossiers-thematiques/la-frontiere-discontinuites-et-dynamiques/articlesscientifiques/maroni-frontiere-guyane-suriname

BRGM, L'or en Guyane, Cayenne, BRGM, 1995.

de Theije, Mario ; Heemskerk, Marieke, "Moving Frontiers in the Amazon : Brazilian Small-Scale Gold Miners in Suriname", European Review of Latin American and Caribbean Studies, n87, 2009, p. 5-25.

de Theije, Mario ; Heemskerk, Marieke, "O Garimpo de ouro na sociedade maroon contemporânea no Suriname", Teoria e Cultura, vol. 9 n², 2014, p. 33-39.

Farruggia, Flora, La gouvernance du secteur minier légal en Guyane, mémoire de stage du projet GUYINT, Paris, INRA, CNRS, 2019.

Granger, Stéphane, La Guyane et le Brésil, ou la quête d'intégration continentale d'un département français d'Amérique, les enjeux d'une cooperationtransfrontaliere nord-sud, Thèse de doctorat à l’Université Paris 3, 2012.

Jacob, Téo, De la conservation en Amazonie: Norme environnementale et démocratie territoriale à l'heure $d u$ « Faire mieux avec moins ", thèse de doctorat de l'Ecole des Hautes Études en Sciences Sociales, 2018.

Le Tourneau, François-Michel ; Greissing, Anna, Le Jari, géohistoire d'un grand fleuve amazonien, Rennes, PUR, 2013.

Le Tourneau, François-Michel, L'Amazonie, histoire, géographie, environnement, Paris,CNRS Editions, 2019.

Le Tourneau, François-Michel, Chercheurs d'or : l'orpaillage clandestin en Guyane française, Paris, CNRS Editions, 2020.

Le Tourneau, François-Michel, "Le « système garimpeiro » et la Guyane : l'orpaillage clandestin contemporain en Amazonie française”, Les Cahiers d'Outre-Mer, n²82, 2020, 263-290.

Mam Lam Fouck, Serge, La Guyane française au temps de l'esclavage, Matoury, Ibis Rouge, 1999.

Mathis, Armin ; Chaves de Brito, David ; Brüseke, Franz, Riqueza Volátil, a mineração de ouro na Amazônia, Belém, CEJUP, 1997.

May, Axel, Guyane française l'or de la honte, Paris,Calmann-Lévy, 2007.

Oliveira, Rafael da Silva, Mobilidades transgressoras, geografias ignoradas: Itinerários e emaranhamentos envolvendo territorialidades de garimpeiros no Suriname, thèse de doctorat de l'université de São Paulo, https://doi.org/10.11606/T.8.2014.tde-12022014-130153, 2014.

Orru, Jean-François, « L'activité aurifère dans la commune de Maripasoula, impact écologique et humain », Journal d'agriculture traditionnelle et de botanique appliquée, n¹-2, 1998. p. 147-166.

Orru, Jean-François, « Annexe 12. Inventaire historique de l'activité d'orpaillage en Guyane française ». In Jean-Pierre Carmouze, Marc Lucotte, et Alain Boudou (Éds.), Le mercure en Amazonie : Rôle de l'homme et de l'environnement, risques sanitaires, Marseille, IRD Éditions, 2001, p. 409-424.

Petot, Jean, L'or de Guyane, son histoire, ses hommes. Paris,éditions Caribéennes, 1986.

Petot, Jean, Histoire contemporaine de l'or en Guyane, Paris,l'Harmattan, 1993.

Heemskerk, Marieke ; Jacobs, Eelco ; Pratley, Pierre, The final frontier of Malaria control and elimination Assessment of mobile migrant population size, demographics, turnover, movement, and priority health needs, KIT Technical report, Amsterdam,Royal Tropical Institute, 2021. 
Salomão, Enzo, "O ofício e a condição de garimpar" in Gerôncia Albuquerque Rocha (org.), Em Busca do ouro. Garimpos e garimpeiros no Brasil, São Paulo, Marco Zero, 1984, p. 35-86.

Silva, Gutemberg ; Granger, Stéphane ; Le Tourneau, François-Michel, « Mudanças e permanências na circulação na fronteira entre Brasil e Guiana Francesa (França) ", Mercator, vol. 18, e18018, 2019, doi: 10.4215/rm2019.e18018.

Souza de Mello, Laura, Desclassificados do ouro: a pobreza mineira no século XVIII, Rio de Janeiro, Graal, 1982.

Strobel, Michèle-Baj, Les gens de l'or, Paris, Plon, 2019.

Tedesco, Leticia da Luz, No trecho dos garimpos mobilidade, gênero e modos de viver na garimpagem de ouro amazônica, thèse de doctorat Université d'Amsterdam, 2015.

Théry, Hervé, Rondônia, mutations d'un Territoire fédéral en Amazonie Brésilienne, Thèse de doctorat, Université Panthéon-Sorbonne - Paris I, 1976.

Wanderley, Luiz Jardim de Moraes, Geografia do ouro na Amazônia brasileira : Uma análise a partir da porção meridional, Thèse de doctorat. Université fédérale de Rio de Janeiro, 2015.

\section{NOTES}

1. Il faudra attendre 1930 pour voir une tentative de reprise en main de la région avec la création d'une subdivision administrative spéciale (le territoire de l'Inini). Faute de moyens, cette tentative n'aura cependant aucun résultat pratique sur l'activité aurifère ou sur le contrôle du territoire.

2. Celle-ci est liée à la fin du gold exchange standard par les États-Unis en 1971. Ce système fixait en effet une conversion fixe de l'or en monnaie, et ce prix fixe n'était pas très avantageux au regard des coûts de production. Avec son abandon, l'or devient une marchandise dont le cours peut fluctuer en fonction de l'offre et de la demande. Les chocs pétroliers des années 1970 vont contribuer à le faire monter, l'or étant considéré comme une valeur de réserve et donc très demandé dans les moments de crise.

3. Ces titres étaient normalement uniquement des autorisations de prospection individuelle, mais ils étaient en pratique utilisés comme des concessions.

4. Même si on est entré irrégulièrement, il est possible d'obtenir une injonction à quitter le territoire surinamais et de l'utiliser pour prendre l'avion, mais cela ne peut se faire que sur présentation de documents d'identité.

5. L'orpaillage peut être légalisé par des concessions, mais il est aussi laissé à la discrétion des communautés bushinenguées qui occupent la plus grande partie des territoires à proximité du Maroni. Celles-ci tolèrent les chantiers en échange d'une redevance qui est en général de $10 \%$ de la production.

6. Sorte de large plat en métal au fond conique permettant d'extraire l'or des sédiments les plus lourds par gravité.

7. Populations issues du marronnage des esclaves dans l'intérieur du Suriname

8. Si les deux pays ont avancé sur la question du Maroni, il demeure encore un contesté frontalier plus au sud, le Suriname revendiquant le territoire situé entre les rivières Marouini et Litani (Blancodini P., 2019).

9. La plupart de ces virements sont effectués par l'intermédiaire de banques brésiliennes en ligne comme N.U. Il ne s'agit pas de virements internationaux à proprement parler (qui apparaîtraient dans les statistiques des banques centrales), mais de systèmes de compensations opérés par des acteurs qui participent du circuit occulte des devises au Brésil. 
10. Les $2 \%$ restant proviennent d'autres pays des Caraïbes, principalement Saint Domingue, Cuba et le Venezuela. On trouve aussi quelques très rares Bushinengués du Suriname.

11. On parle ici du risque lié à l'action des autorités. D’autres risques sont considérés comme presque inévitables, comme celui d'être rançonné lorsque l'on revient en ville avec son or. Pour cela les orpailleurs essayent de l'envoyer au fur et à mesure, ce qui diminue les risques.

12. Mis à part les tragiques événements de Dorlin en 2012, où une bande armée a ouvert le feu, tuant deux militaires du $9^{\mathrm{e}}$ RIMA et blessant grièvement deux gendarmes, les affrontements dépassent rarement des invectives ou des coups de feu en l'air.

13. Le mercure est une substance surveillée et son usage en principe restreint à des opération industrielles précises, ou bien à un usage en dentisterie.

\section{RÉSUMÉS}

La Guyane française est exploitée par plusieurs milliers d'orpailleurs clandestins, qui en arrachent chaque année entre cinq et dix tonnes d'or, exportées en contrebande vers le Suriname et le Brésil. Presque exclusivement brésiliens, ces mineurs informels, appelés garimpeiros, déploient en Guyane le système économique, culturel et social qui fait leur spécificité au Brésil, et qui couvre aussi le Suriname. Au cœur de ce système se trouve une logique territoriale propre aux orpailleurs, qui articule entre elles les différentes régions d'exploitation à l'échelle de l'Amazonie et du plateau des Guyanes sous la forme d'un réseau au fonctionnement transnational, ou plutôt «a-national» car il ne prend tout simplement pas en compte les frontières autrement que comme d'éventuels obstacles à contourner. Les garimpeiros approchent les différents territoires en fonction d'une grille de lecture qui leur est propre, dans laquelle la richesse des gisements ou la pression des autorités sont des facteurs beaucoup plus forts que l'appartenance d'un territoire à tel ou tel pays, réduisant de ce fait les frontières à de simples contraintes matérielles. Fondé sur une recherche de quatre années sur le phénomène de l'orpaillage clandestin au Brésil, l'article montre comment le « système garimpeiro » se déploie en Guyane en exploitant la difficulté des contrôles frontaliers, tant sur les façades fluviales que sur la façade maritime, et comment l'univers des camps d'orpaillage de Guyane est un prolongement de ceux du Brésil et du Suriname avec lesquels il est profondément articulé.

A Guiana francesa segue explorada clandestinamente por milhares de garimpeiros que extraem e contrabandeam entre cinco e dez toneladas de ouro a cada ano para o o Suriname ou o Brasil. Quase todos brasileiros, esses garimpeiros reproduzem no território francês o sistema econômico, social e cultural que fa a sua especificidade no Brasil, e que extenderam também no Suriname. Tal sistema encontra-se baseado numa lógica territorial própria que articula as diversas regiões de garimpo entre si na forma de uma rede cujo funcionamento é transnacional ou, melhor, a-nacional pois ele não considera as fronteiras internacionais além de seram obstáculos que devem ser contronados. Os garimpeiros avaliam os diferentes territórios em função de critérios próprios nos quais a riqueza das jazidas ou a pressão das autoridades locais são elementos muito mais importantes que o pertencimento das áreas a tal ou tal país, reduzindo assim as fronteiras a meras contingências materiais. Baseado em cima de quatro anos de estudo da garimpagem ilegal na Guiana francesa, o artigo mostra com o « sistema garimpeiro » extendese no território francês valendo-se da dificuldade de contrôle das fronteiras fluviais ou 
marítimas, assim como o universo dos garimpos da Guiana aparece como uma extensão dos garimpos do Brasil e do Suriname, com os quais tem profundas articulações.

The French territory of Guiana is currently exploited illegally by thousands of small-scale artisanal gold miners who extract each year between 5 and 10 tons of gold and smuggle them into Suriname and Brazil. Almost exclusively Brazilian and called garimpeiros, these miners deploy in French Guiana the economic, social and cultural system that makes their specificity in Brazil, and that covers Suriname as well. This system is grounded on a specific territorial logic which articulates all the mining territories of the Brazilian Amazon and of the Guiana plateau in the form of a transnational or, rather, an a-national network in which borders are no more than obstacles that are hardly impossible to overcome. The garimpeiros approach the different territories in function of their own evaluation grid where a region richness in gold or the pressure by authorities to prohibit their activities are much more relevant than the country the area they consider belongs to, thus reducing borders to mere material contingencies. Based on four years of extensive research about illegal gold mining in French Guiana, this paper shows how the "garimpeiro system" is deployed in the French territory and exploits the difficulty of border control, both on rivers and at sea. It also analyzes how this system links illegal mining areas in French Guiana with the ones in Brazil and in Suriname.

\section{INDEX}

Mots-clés : Orpaillage, Guyane, Frontières, Suriname, Immigration illégale

Palavras-chave : Garimpo, Guiana francesa, fronteiras, Suriname, imigração ilegal

Keywords : Gold mining, French Guiana, borders, Suriname, Illegal immigration

\section{AUTEUR}

\section{FRANÇOIS-MICHEL LE TOURNEAU}

François-Michel Le Tourneau est géographe et directeur de recherche au CNRS, affecté à l'IRL 3157 iGLOBES (CNRS / University of Arizona). Spécialiste de l'Amazonie brésilienne, il mène depuis 2016 des travaux sur l'orpaillage clandestin en Guyane française. francois-michel.letourneau@cnrs.fr 